Case report

\title{
Post-mortem computed tomography angiography and forensic necropsy of a brown howler monkey: A case report
}

\author{
Ana Carolina Brandão de Campos Fonseca Pinto ${ }^{\mathrm{a}}$, Mara Rita Rodrigues Massad ${ }^{\mathrm{b}}$, \\ Laila Massad Ribas, ${ }^{\mathrm{b}, *}$, Carina Outi Baroni ${ }^{\mathrm{a}}$, Tália Missen Tremori ${ }^{\mathrm{b}}$, Sérvio Túlio Jacinto Reis ${ }^{\mathrm{b}}$, \\ Noeme Sousa Rocha ${ }^{b}$ \\ a Surgery Department, School of Veterinary Medicine and Animal Science, University of São Paulo, São Paulo, Brazil \\ b Department of Veterinary Clinical Sciences, School of Veterinary Medicine and Animal Science, São Paulo State University, Botucatu, Brazil
}

\section{A R T I C L E I N F O}

\section{Keywords:}

Forensic pathology

Post-mortem diagnosis

Primates

Radiology

\begin{abstract}
A B S T R A C T
The purpose of this case report was to compare, in a brown howler monkey (Alouatta guariba clamitans), the findings of the post-mortem computed tomography (PMCT) with the forensic necropsy, and also to describe a successful post-mortem computed tomography angiography (PMCTA) study. Post mortem CT images revealed two thyroid cartilage fractures that were not seen at the forensic necropsy. The PMCTA was a feasible technique, but did not change the final diagnosis in this case. The PMCT findings were considered crucial to determine the cause of the disability of the animal, and this disability was believed to contribute to the cause of death. In conclusion, PMCT added relevant information to the establishment of the final diagnosis, and can be considered an important adjuvant tool to the veterinary pathology.
\end{abstract}

\section{Introduction}

In veterinary medicine post-mortem radiology is a new field that has grown up fast and has helped the pathologists to achieve a more detailed evaluation of the deceased [1,2]. Nowadays, although the fact that post-mortem computed tomography (PMCT) and post-mortem computed tomography angiography (PMCTA) are still not considered part of the widely routine in veterinary pathology, these techniques have being gradually introduced to improve the post-mortem diagnosis [3-5].

In trauma cases, PMCT is one of the best post-mortem modalities to identify small fractures and the presence of gas in cavities or in the vascular system [6-8]. Even though PMCTA is a well-established technique [9] there is no report of its use in small primates. The PMCTA exam consists in the injection of a contrast into the vascular system, and can enhance the quality of the images and offer a better understanding of the trauma when there is a vascular leaking or an organ rupture [10].

In wild animals, one of the major indications to perform a forensic necropsy is the identification of lesions caused by human cruelty, motor vehicles or other animals [11]. The lack of previous information and the difficulties to clinically evaluate wild animals create a more challenging scenario to the veterinarian in the establishment of the cause of death.
Therefore, the purpose of this case report was to compare the findings of the PMCT with the forensic necropsy and also to describe a successful PMCTA in a brown howler monkey (Alouatta guariba clamitans).

\section{CASE}

A wild brown howler monkey (Alouatta guariba clamitans) (4.5 kg) was found and rescued in the north zone of São Paulo city, State of São Paulo-Brazil by the Environmental Military Police and sent to the Department of Parks and Green Areas (DEPAVE). At an external examination, the animal presented a deep injury in the left hind limb infested by myiasis and with exposed musculature, vessels and tendons. It was considered an old animal, between 15 and 20 years old, due to the worn teeth and a periodontal disease (Fig. 1). Because the extension of the injury and its advanced age the animal was euthanized.

The cadaver was freezed at $-17^{\circ} \mathrm{C}$ for 30 days, until it was sent to the veterinary hospital where the PMCT and PMCTA were performed. Images were acquired pre and post contrast injection with the cadaver positioned in dorsal recumbency, using a Philips 16-slice multidetector scanner MX800 (Philips, Cleveland, OH, USA). The scan parameters were $120 \mathrm{kVp}, 350 \mathrm{mAs}$, the slice thickness ranged from $1 \mathrm{~mm}$ (head and lung parenchyma) to $2 \mathrm{~mm}$ (abdomen and mediastinum), the slice interval ranged from

\footnotetext{
* Correspondence to: Department of Veterinary Clinical Sciences, School of Veterinary Medicine and Animal Science, São Paulo State University, Rua Prof. Doutor Walter Mauricio Correa s/n - Caixa Postal 560, zip code 18618-681 - Botucatu, SP.

E-mail address: lailamassad@gmail.com (L. Massad Ribas).
} 


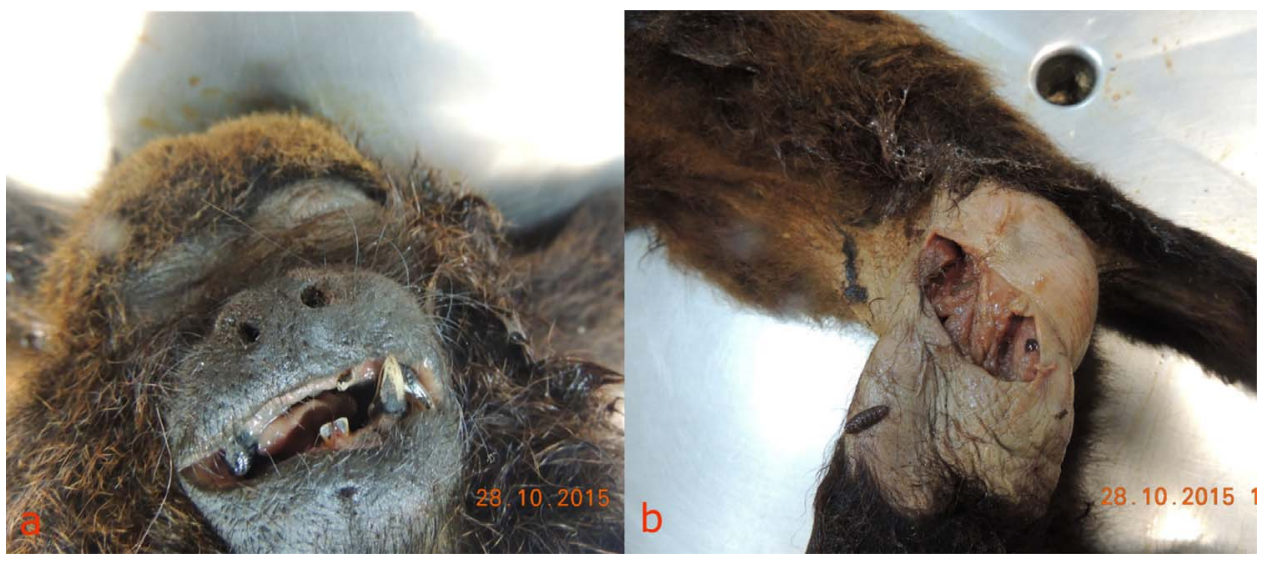

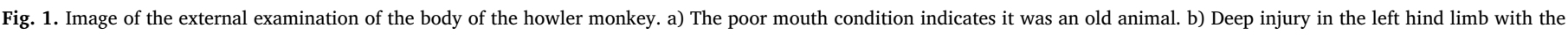
exposure of the musculature, vessels and tendons with myiasis infestation.

$0,5 \mathrm{~mm}$ (head and lung parenchyma) to $1 \mathrm{~mm}$ (abdomen and mediastinum).

In order to acquire the post contrast images, a $98 \mathrm{~mL}$ of a mixture solution in a ratio of 1:20 of an ionized water-soluble contrast medium, iohexol $(300 \mathrm{mg} / \mathrm{mL})$, and polyethylene glycol 300 was prepared. This volume represented approximately $28 \%$ of the blood volume of the animal. To inject the mixture, a dissection of the femoral artery and vein was done by unilateral inguinal incision. The retrograde cannulation of the femoral artery and the antegrade cannulation of the femoral vein were done both with a 6-French cannula introduced about $7 \mathrm{~cm}$ inside the vessels and connected to an injection pump (Medrad Vistron CT, Indianola, PA, USA). The cannulas were previously filled with the mixture to prevent injection of air bubbles. Half of the mixture was injected in each phase (arterial and venous) with a pressure gradient of 300 PSI and a flow rate of $30 \mathrm{~mL} / \mathrm{min}$ (Fig. 2).

The PMCT images revealed two fractures of thyroid cartilage, one to the right side, associated with mild soft tissue swelling and one to the left that was located more dorsally (Fig. 3). The lack of some incisive tooth and periapical lyses in some pre-molar tooth were noted. The images revealed also air-fluid levels in the maxillary sinus (more evident in the right side) and the hyoid bone. There was also noted skin, subcutaneous and muscular laceration in the left hind limb, but no bone injuries were detected (Fig. 4). In the thoracic PMCT were seen pleural effusion and alveolar pattern with air bronchograms associated with ground glass attenuation, more evident in the left side (Fig. 5).

Images also presented signs of decomposition of the body as gas in the right parietal region of the brain and in the abdominal cavity. In the PMCTA mild leakage of the contrast in the parietal and occipital region of the left hemisphere of the brain was observed.

After the PMCT procedure, the body was sent to a veterinary pathologist that performed the forensic necropsy blinded to results of the computed tomography exam. At external examination it was possible to observe that the left hind limb lesion was limited to skin and muscles, with no bone injuries. At macroscopic analysis, lungs, liver, kidneys, spleen and brain were in autolysis and there was foamy trachea secretion, compatible with pulmonary oedema. Forensic necropsy did not revealed the brain or free abdominal gas accumulation neither the thyroid cartilage fractures.

\section{Discussion}

This case report describes a PMCT and a successful PMCTA performed in a brown howler followed by conventional necropsy. The images revealed two sites of fractures of the thyroid cartilage that were not seen in the conventional necropsy. In human medicine it is very common to detect small fractures with post-mortem computed tomo-

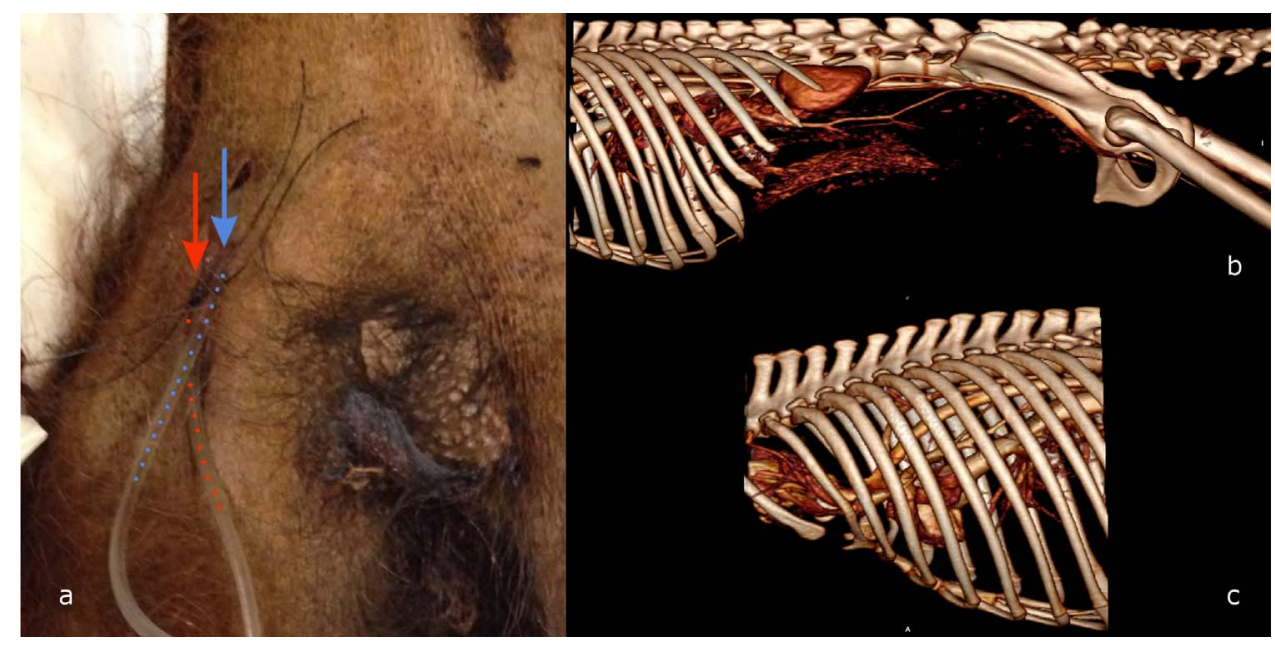

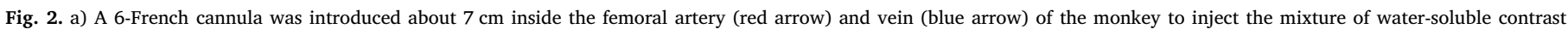

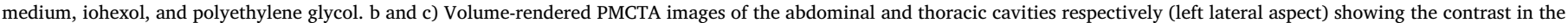
vessels, kidney and heart. (For interpretation of the references to color in this figure legend, the reader is referred to the web version of this article). 


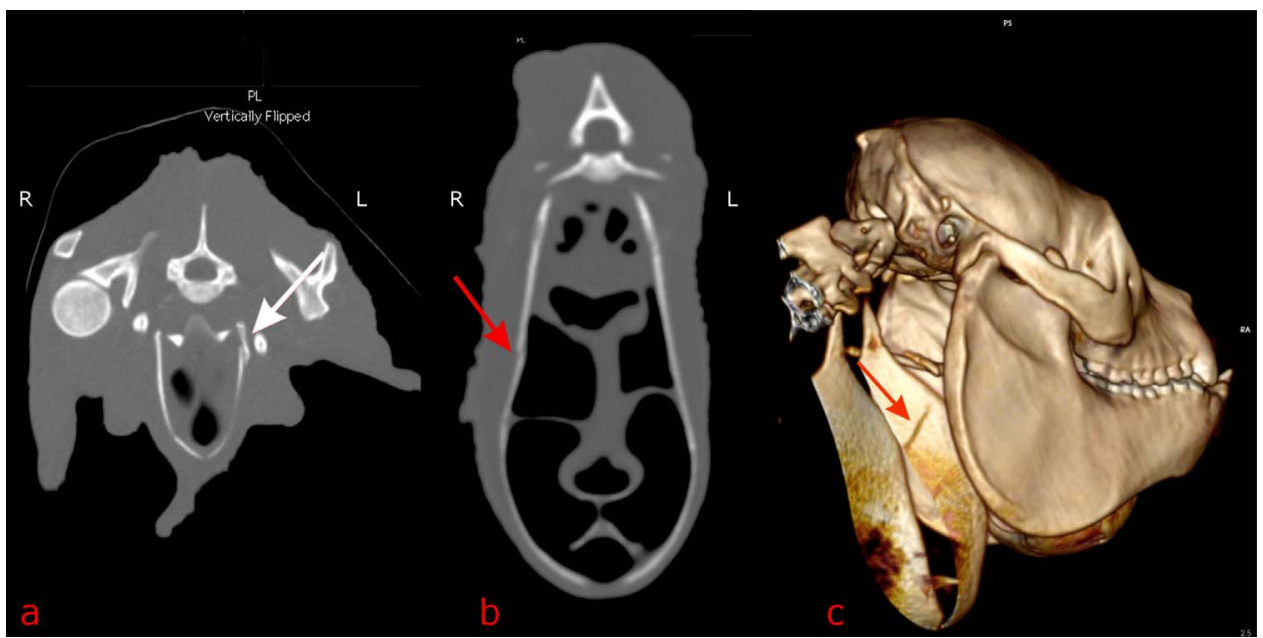

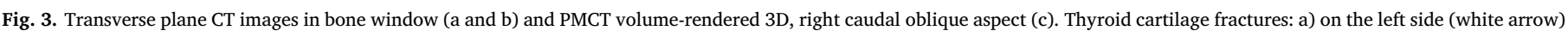

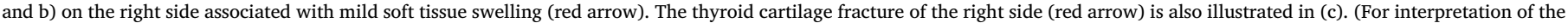
references to color in this figure legend, the reader is referred to the web version of this article).

graphy that are not diagnosed by the conventional necropsy. This is one of the major indications for the PMCT especially in forensic cases [12-14].

Neck fractures in humans are strongly related to manual strangulation or hanging [15], but rarely related to blunt neck trauma [16]. In this howler monkey, there were no signs of facial venous congestion, lymphatic oedema or ophthalmologic petechiae, which are mainly related to strangulation [17]. Hence, it is possible to believe that thyroid cartilage fractures were due to a blunt neck trauma and the extensive injury in the left hind limb was possibly due to an animal biting.

Howler monkeys have a pneumatized hyoid bone to amplify the sound of the howl, and this vocalization is considered a very important way of communication, especially by males and during the reproduction season [18]. Furthermore, there are evidences that another function of the howl is to defend food resources from other monkeys [19]. Therefore, the thyroid cartilage fractures associated with the lack of some teeth and periapical lyses in some pre-molar tooth could be a reasonable explanation for the poor overall condition of this animal and

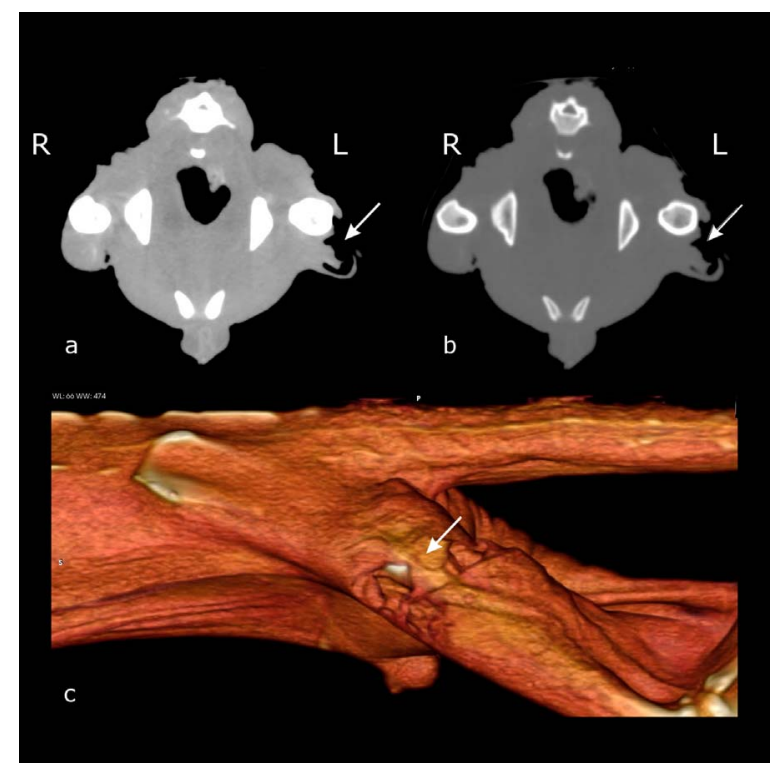

Fig. 4. Transverse CT images in soft tissue (a) and bone window (b) and 3D volumerendered CT image (c) illustrating the skin, subcutaneous and muscular laceration in the left hind limb without bone injuries. also might explain its increased risk to suffer other animal attack. The detection of the thyroid cartilages fractures reinforces the importance of performing the PMCT studies in this cadaver, since these fractures were not identified at the conventional necropsy.

The minimum amount of pleural effusion, the pneumocephalus, the presence of free gas in the abdominal cavity and the leakage of the contrast in the brain can be related to post-mortem features as autolysis and decomposition starts immediately upon death [20]. Besides the forensic necropsy could not reveal these findings, the autolysis process observed of the organs confirmed the decomposition of the body.

The ground glass attenuation is a common PMCT finding and could be related to internal livor mortis, oedema, pneumonia or aspiration [21]. Images did not reveal the foamy trachea secretion, observed in the forensic necropsy. This last specific finding could confirm that this lung attenuation was due to the oedema of the euthanasia procedure and not only to a post-mortem process.

The use of post-mortem images in animals can contribute not only to veterinary medicine, but also to medical research. Being the howler a small primate, this specimen can be considered an experimental model for PMCTA in children, mainly when considering the difficulty of vessels access for the contrast injection in those small individuals. In human PMCTA, the contrast is injected by a roller pump [10]. In our study we used a conventional tomography injection pump, which has the capability to inject up to $200 \mathrm{~mL}$, a sufficient volume for small primates.

To the best of our knowledge, this was the first PMCT, PMCTA reported in a howler monkey, and their results could add important information to allow the establishment of the possible cause of the disability or death of the animal. Considering the comparison between PMCT and necropsy, both techniques demonstrated injuries one complementing the other. To conclude, the inclusion of the PMCT added relevant information to the establishment of the final diagnosis, being then considered an important adjuvant tool to the veterinary pathology.

\section{Declaration of interest}

The authors report no conflicts of interest. The authors alone are responsible for the content and writing of the paper.

\section{Acknowledgements}

We thank the Environmental Military Police and the Department of Parks and Green Areas (DEPAVE) for their contribution. This work was 

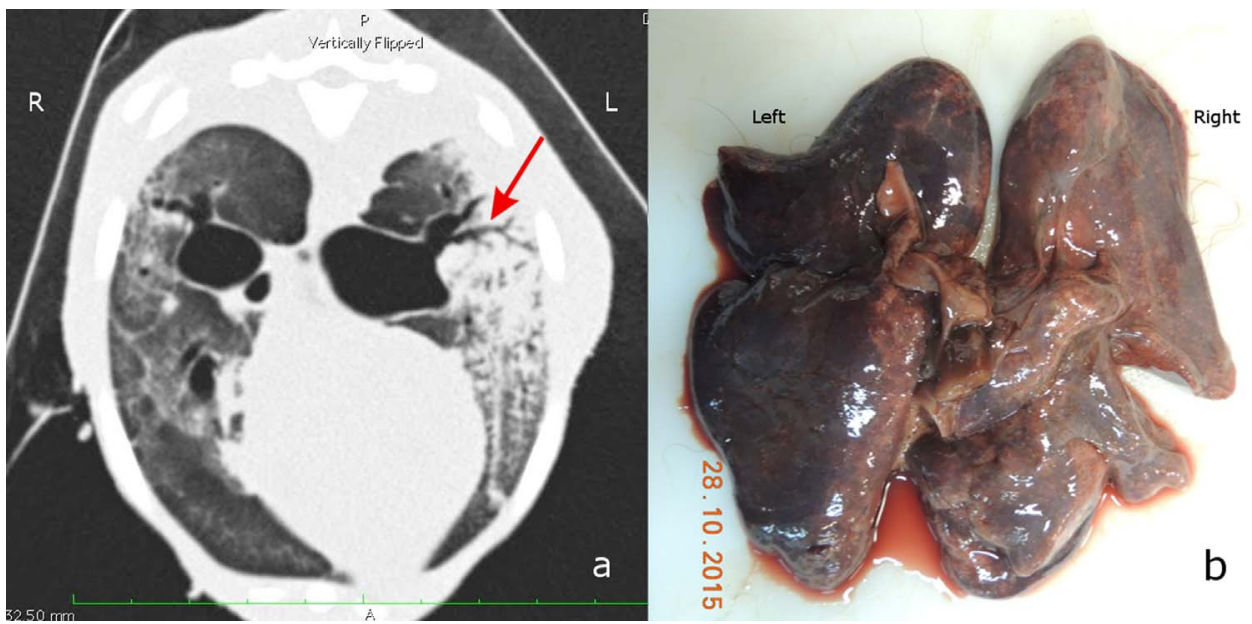

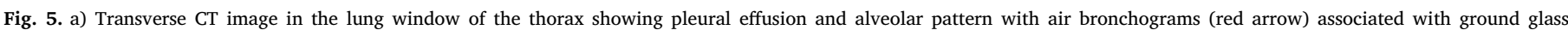

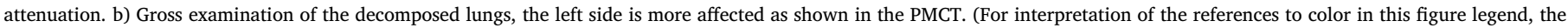
reader is referred to the web version of this article).

supported by Coordenação de Aperfeiçoamento de Pessoal de Nível Superior - CAPES, Edital Ciências Forenses 25/2014. Process n. 99999.000199/2016-08.

\section{References}

1 E. Watson, H.G. Heng, Forensic radiology and imaging for veterinary radiologists, Vet. Radiol. Ultrasound (2017) 1-14.

2 A.C.B.C.F. Pinto, M.R.R. Massad, L.M. Ribas, C.O. Baroni, T.M. Tremori, S.T.J. Reis, N.S. Rocha, Complete cardiac and bronchial avulsion in a dog: post-mortem computed tomography and forensic necropsy analysis, J. Forensic Radiol. Imaging (2017) (in press)

3 M.J. Thali, B.P. Kneubuehl, S.A. Bolliger, A. Christe, U. Koenigsdorfer, et al., Forensic veterinary radiology: ballistic-radiological 3D computertomographic reconstruction of an illegal lynx shooting in Switzerland, Forensic Sci. Int. 171 (2007) 63-66.

4 K.J. Lee, M. Sasaki, A. Miyauchi, M. Kishimoto, J. Shimizu, et al., Virtopsy in a red kangaroo with oral osteomyelitis, J. Zoo. Wildl. Med. 42 (2011) 128-130.

5 R.M. Martinez, U. Hetzel, M.J. Thali, W. Schweitzer, Cat CAT-scan: postmortem imaging and autopsy of two cats, J. Forensic Radiol. Imaging 3 (2015) 80-86.

6 F. Makhlouf, V. Scolan, G. Ferretti, C. Stahl, F. Paysant, Gunshot fatalities: correlation between post-mortem multi-slice computed tomography and autopsy findings: a 30months retrospective study, Leg. Med. 15 (2013) 145-148.

7 B.A. Hoey, J. Cipolla, M.D. Grossman, N. McQuay, P.R. Shukla, et al., Postmortem computed tomography, "CATopsy", predicts cause of death in trauma patients, J. Trauma 63 (2007) 979-985.

8 T. Zerbini, L.F. Silva, A.C. Ferro, F.U. Kay, E. Amaro Junior, et al., Differences between postmortem computed tomography and conventional autopsy in a stabbing murder case, Clinics 69 (2014) 683-687.

9 S.G. Ross, S.A. Bolliger, G. Ampanozi, L. Oesterhelweg, M.J. Thali, et al., Postmortem CT angiography: capabilities and limitations in traumatic and natural causes of death,
RadioGraphics 34 (2014) 830-846.

10 S. Ross, D. Spendlove, S. Bolliger, A. Christe, L. Oesterhelweg, et al., Postmortem whole-body CT angiography: evaluation of two contrast media solutions, Am. J. Roentgenol. 190 (2008) 1380-1389.

11 G. Wobeser, Forensic (medico-legal) necropsy of wildlife, J. Wild Dis. 32 (1996) 240-249.

12 A. Christe, S. Ross, L. Oesterhelweg, D. Spendlove, S. Bolliger, et al., Abdominal trauma: sensitivity and specificity of postmortem noncontrast imaging findings compared with autopsy findings, J. Trauma 66 (2009) 1302-1307.

13 C. Chevallier, F. Doenz, P. Vaucher, C. Palmiere, A. Dominguez, et al., Postmortem computed tomography angiography vs. conventional autopsy: advantages and inconveniences of each method, Int. J. Leg. Med. 127 (2013) 981-989.

14 F.Z. Mokrane, F. Savall, C. Rérolle, A. Blanc, P. Saint Martin, et al., The usefulness of post-mortem CT angiography in injuries caused by falling from considerable heights: three fatal cases, Diagn. Interv. Imaging 95 (2014) 1085-1090.

15 P.P. Mukhopadhyay, Predictors of hyoid fracture in hanging: discriminant function analysis of morphometric variables, Leg. Med. 12 (2010) 113-116.

16 V.D. Khokhlov, Trauma to the hyoid bone and laryngeal cartilages in hanging: review of forensic research series since 1856, Leg. Med. 17 (2015) 17-23.

17 S.K. Verma, S. Lal, Strangulation deaths during 1993-2002 in East Delhi (India), Leg. Med. 8 (2006) 1-4.

18 H.L. Farmer, A.B. Plowman, L.A. Leaver, Role of vocalisations and social housing in breeding in captive howler monkeys (Alouatta caraya), Appl. Anim. Behav. Sci. 134 (2011) 177-183.

19 S.V. Belle, A. Estrada, P.A. Garber, The function of loud calls in black howler monkeys (Alouatta pigra): food, mate, or infant defense? Am. J. Primatol. 76 (2014) 1196-1206.

20 L. Saba, J.S. Suri, Multi-detector CT imaging: abdomen, pelvis, and CAD applications, CRC Press, Boca Raton, 2014.

21 A. Christe, P. Flach, S. Ross, D. Spendlove, S. Bolliger, et al., Clinical radiology and postmortem imaging (Virtopsy) are not the same: specific and unspecific postmortem signs, Leg. Med. 12 (2010) 215-222. 